Pak. j. sci. ind. res. Ser. B: biol. sci. 2020 63B(2) 86-92

\title{
Antioxidant and Antidiabetic Potential of Saponin Fraction Isolated from Moringa oleifera Leaves
}

\author{
Fatma Hussain* and Fatima Ikram \\ Clinico-Medical Biochemistry Laboratory (CMBL), Department of Biochemistry, Faculty of Sciences, \\ University of Agriculture, Faisalabad-38040, Pakistan
}

(received February 12, 2018; revised October 2, 2018; accepted October 10, 2018)

\begin{abstract}
To assess total phenolic contents, total flavonoid contents (TPC, TFC), DPPH scavenging and antidiabetic (antiglycation and alpha amylase hindrance) activities of Moringa oleifera (MO) leaves, bioassay guided fractionation was used with thin layer chromatography profile to isolate saponin fraction (SF). The TPC and TFC were 9.7-12.8 GAE and 5.3-9.3 CE (g/100 g dry weight), respectively and aqueous and methanol fractions were more effective. DPPH scavenging activity ranged $69.5-83.1 \%$. SF displayed noteworthy antioxidant ability $(\mathrm{p}<0.046)$ and it was leading inhibitor of glycation $(\mathrm{p}<0.041)$ after $\mathrm{MeOH}$ with $48.1 \%$ restriction. Enzyme inhibition by MO fractions was negligible (3.4-9.2\%) and it was less impressive by SF as compared to $\mathrm{MeOH}$ extract. Percent reduction in enzyme activity by SF was nonsignificant $(\mathrm{p}>0.198)$, nullifying its concentration dependent impact.
\end{abstract}

Keywords: Moringa oleifera, antioxidant activity, antidiabetic activity, saponin fraction

\section{Introduction}

Pharmacological exploitation of natural compounds is promising at betterment of treatment of a variety of diseases. Medicinal plants such as Moringa oleifera attract increasing interest in the beneficial management of diabetes mellitus owing to remarkable antioxidant properties. M. oleifera Lam. has been an important plant since ancient times, both for its nutritional and medicinal uses. Recently, several therapeutic potentials of M. oleifera such as antioxidant, antimicrobial, anti-inflammatory, anticancer and antidiabetic have been studied. Apart from these effects, the potential therapeutic implications in treatment of endocrine, circulatory, neural, respiratory and digestive disorders are well reported (Jung, 2014; Sahoo et al., 2014).

M. oleifera leaves have been used as an important source to treat downstream complications related with elevated serum creatinine (Sahoo et al., 2014). Protecting effect of $M$. oleifera against carcinogenesis, cataract, inflammation and diabetes mellitus are clearly indicated (Kurmi et al., 2014; Waterman et al., 2014; Yassa and Tohamy, 2014; Bharali et al., 2003). M. oleifera seeds hydroalcoholic extract and its chloroform fraction were effective to treat experimental colitis (Minaiyan et al., 2014). Chemopreventive, anti-leukemic and antipyretic activities of $M$. oleifera strongly supports its ethnopharmacological uses (Ahmad et al., 2014; Akanni et al., 2014).

*Author for correspondence; E-mail: fatmauaf@yahoo.com
Oxidative stress is mainly involved in the development of various degenerative ailments, such as cardiovascular disease and diabetes mellitus. M. oleifera could be beneficial by reducing oxidative stress and especially hyperglycemia that leads to formation of advanced glycation endproducts (AGE). Moreover, M. oleifera may decrease lipid synthesis and cholesterol and can act as antileishmanial agent (Kaur et al., 2014; Ngamukote et al., 2014; Sangkitikomol et al., 2014).

Saponins, the natural glycosides of steroid or triterpene are pharmaceutical and nutraceutical agents mostly present in plant leaves and roots. These possess hypocholesterolemic, antidiabetic, anticoagulant, hepatoprotective, anticarcinogenic, hypoglycemic, neuroprotective, immunomodulatory, anti-inflammatory, antimicrobial and antioxidant activity, leading to significant concentration on their benefit to be used as vaccine adjuvants (Koneri et al., 2014; Thao et al., 2014; Astuti et al., 2011; Sun et al., 2009; Rao and Gurfinkel, 2000). Estimation of total saponins in MO is a routine practice that needs to be more elaborated in terms of antioxidant and antidiabetic efficacy of isolated saponins. Phytochemicals are isolated by extraction with numerous polarity based solvents and these constituents exhibit variation in their chemistry and ultimately solubility in specific solvent (Sultana et al., 2009). Despite the natural abundance and widespread use, data on biological activities of MO leaves in different solvents and the isolated saponins is limited. The purpose of the present 
study was to investigate $M$. oleifera leaves solvent fractions and isolated saponins fraction for antioxidant and antidiabetic activities.

\section{Materials and Methods}

Collection, identification and preparation of plant material. Samples collected from local Botanical garden were authenticated by a botanist. Leaves were washed, shade dried and ground to coarse powder. Powdered material was initially extracted with methanol at $25^{\circ} \mathrm{C}$ for 4 days. Methanolic extracts were evaporated and resulting viscous greenish residues were partitioned into fractions by separating funnel. Fractionation was done into $n$-hexane (120 g), $n$-butanol (40 g), ethyl acetate (55 g), chloroform (60 g) and aqueous (85 g) fractions.

Phytochemical analysis. Phytochemical screening was accomplished for the detection of active components like saponins, tannins, alkaloids, terpenoids and flavonoids (Krishnaiah et al., 2009).

Chromatographic isolation of saponin. The $n$-butanol fraction with optimal saponin concentration was further processed by means of thin layer chromatography (TLC) using silica gel G60 to validate and isolate saponins as described previously (Wagner and Bladt, 1996). Three spots that matched the reference $\mathrm{R}_{\mathrm{f}}$ values of saponins were scratched from TLC plates, centrifuged and supernatant was dried to obtain pure saponin fraction.

Antioxidant components. Total phenolic contents (TPC). The amount of phenol contents was measured by Folin-Ciocalteu method, explained previously (Chahardehi et al., 2009). Briefly, solvent fractions and saponin fraction (SF) were incorporated in $1 \mathrm{~mL}$ DMSO (dimethyl sulfoxide) and $1 \mathrm{~mL}$ Folin- Ciocalteu reagent (10\%). $3 \mathrm{~min}$ later, $3 \mathrm{~mL} \mathrm{Na}_{2} \mathrm{CO}_{3}(1 \% \mathrm{w} / \mathrm{v})$ was added and incubated at room temperature for $2 \mathrm{~h}$. The results were reported as gallic acid equivalent ( $\mathrm{g}$ gallic acid/100 g dry weight) after measuring absorbance $(760 \mathrm{~nm})$.

Total flavonoid contents (TFC). Spectrophotometric method (Siddique et al., 2010) was used for this estimation. $0.5 \mathrm{~mL}$ of extracts $(1: 10 \mathrm{~g} / \mathrm{mL})$ was mixed with respective solvent $(1.5 \mathrm{~mL}), 10 \% \mathrm{AlCl}_{3}(0.1 \mathrm{~mL})$, $2.8 \mathrm{~mL}$ distilled water and $0.1 \mathrm{~mL}$ of potassium acetate (1 M). Contents were articulated as g catechin equivalents/100 g dry weight) by taking absorbance at $510 \mathrm{~nm}$.
Antioxidant activity (DPPH scavenging). The fractions were analyzed for their abilities to scavenge DPPH (2, 2-diphenyl 1-1-1-picrylhydrazyl) as described by Souri et al. (2010). $50 \mu \mathrm{L}$ aliquot of some concentrations of each extract was mixed with $5 \mathrm{~mL}$ DPPH methanolic solution of $0.004 \%$. After incubating for $30 \mathrm{~min}$ at room temperature, absorbance was taken at $517 \mathrm{~nm}$. Inhibition percentage was calculated: Radical scavenging $\left(\mathrm{IC}_{50} \%\right)$ $=100 \times$ (Absorbance blank - Absorbance test sample $/$ Absorbance blank). $\mathrm{IC}_{50}$ denote the sample concentration required to scavenge $50 \%$ of DPPH radicals. The experiment was done in triplicates. BHT (Butylated hydroxy toluene) was the standard control.

Antioxidant components and activity were assessed in each sample (extract/fraction/synthetic), which was collected in triplicate and for each collection, three measurements were made. That concludes the use of sixty three samples each for TPC, TFC contents and $\mathrm{DPPH}$ activity assays and statistical analysis.

Antidiabetic evaluation. Antiglycation assay. Antiglycation assay was performed as described before (Ayatollahi et al., 2010) and results were compared to those of the synthetic inhibitor aminoguanidine. Solvent fractions and SF were reconstituted in dimethylsulphoxide (DMSO). Bovine serum albumin (BSA, 10 $\mathrm{mg} / \mathrm{mL})$ and anhydrous glucose $(50 \mathrm{mg} / \mathrm{mL})$ were made in sodium phosphate buffer (67 mM, pH 7). Sample mixture contained equal amounts $(100 \mu \mathrm{L})$ of fraction, BSA and glucose solution. Glycated (positive) control had sodium phosphate buffer instead of plant extract, while blank (negative control) contained BSA and sodium phosphate buffer only. After incubation at 37 ${ }^{\circ} \mathrm{C}$ for 7 days, $100 \mu \mathrm{L}$ of $100 \%$ trichloroacetic acid was added and centrifuged for $4 \mathrm{~min}$ at $4{ }^{\circ} \mathrm{C}$. Pellets containing advanced glycation end product were dissolved in phosphate buffer saline ( $\mathrm{pH} 10)$. Absorbance was noted at $440 \mathrm{~nm}$ and inhibition percentage was calculated, \% inhibition $=100-[(O D($ test $) / O D($ blank $) \times 100]$. Same procedure was repeated after 3 and 5 weeks.

Antiglycation activity was assessed in each sample (extract/fraction/synthetic), which was collected in triplicate for $1^{\text {st }}, 3^{\text {rd }}$ and $5^{\text {th }}$ weeks. For each collection, three measurements were made. That concludes the use of one hundred and eighty nine samples for the antiglycation tests and statistical analysis.

Enzyme inhibition assay. Assay of alpha amylase inhibition was performed according to Apostolidis et al. (2006). Porcine pancreatic $\alpha$-amylase (EC 3.2.1.1) 
was bought from Sigma Chemical Co. Extracts, SF $(500 \mu \mathrm{L})$ and $500 \mu \mathrm{L}$ amylase solution $(0.5 \mathrm{mg} / \mathrm{mL})$ in buffer $\left(0.02 \mathrm{M}\right.$; pH 6.9) were incubated at $25^{\circ} \mathrm{C}$. After $10 \mathrm{~min}, 500 \mu \mathrm{L}$ starch solution (1\%) was added at fixed time intervals and incubated. The reaction was arrested by adding $1 \mathrm{~mL}$ of dinitrosalicylic acid (DNS) reagent, heated in a boiling water bath for $5 \mathrm{~min}$, cooled to room temperature and diluted with $10 \mathrm{~mL}$ distilled water. Absorbance was noted against blank at $540 \mathrm{~nm}$. $\mathrm{IC}_{50}$ $(\%)$ was calculated as: $([\mathrm{Ac}-\mathrm{As} / \mathrm{Ac}]) \times 100$. Where, Ac $=$ the absorbance of control and $\mathrm{As}=$ the absorbance of test samples. For control (negative), buffer was used and positive control was acarbose drug.

Enzyme inhibition was assessed with $0.1,0.3,0.5 \mathrm{~mL}$ extracts/fractions/synthetic inhibitor volume, which was collected in triplicate and for each collection, three measurements were made. That concludes the use of one hundred and eighty nine samples in enzyme inhibitory and statistical analysis.

Statistical analysis. All the results were stated as values of means \pm SD (standard deviation) and analysis of variance (ANOVA) was used to analyze data by SPSS software (SPSS Inc. Chicago, IL, USA; version 15.0) at level of significance $p<0.05$. To assess within group differences due to week wise treatments, repeated measure ANOVA (tukey test) and for multiple comparisons, LSD test (post hoc test) were used at 5\% level of significance (Statistical Software SAS 9.0).

\section{Results and Discussion}

Antioxidant contents and activity. Phytochemicals play an important pharmacological role (Kasolo et al., 2010). This study screened $M$. oleifera leaves fractions especially saponins for antioxidant and antidiabetic activities. Phytochemical screening indicated the availability of imperative chemicals such as tannins, saponins, terpenoids, alkaloids and flavonoids in $\mathrm{MO}$ leaves. SF that appeared on chromatograms had three $\mathrm{R}_{\mathrm{f}}$ values $(0.89,0.90,0.91)$ with green coloured spots. These SF were pooled together into one fraction for the estimation of antioxidant and antidiabetic potentials. TPC and TFC of MO leaf fractions in ranges 9.7-12.8 GAE and 5.3-9.3 CE (g/100 g dry weight) are summarized in Table 1. TPC was significantly higher in methanol than $n$-hexane fraction $(\mathrm{p}<0.034)$. Analysis revealed variations in TPC and TFC of different solvents. Aqueous and methanol were more effective in extracting the highest TPC and TFC, followed by ethyl acetate, chloroform, $n$-butanol and $n$-hexane.

All the fractions had free radical scavenging abilities but to significantly different degrees $(p<0.05)$, in the range 69.5-83.1\% (Table 1). MO methanol extract exhibited highest scavenging activity $(\mathrm{p}<0.037)$. Although SF displayed noteworthy antioxidant ability $(p<0.046)$ as compared to standard BHT, its efficiency was trivial than most of the other extracts. Regarding the solvent partitions, DPPH scavenging activity in descending order was ethyl acetate $>$ chloroform $>n$ butanol $>\mathrm{SF}>n$-hexane. In terms of $\mathrm{IC}_{50}$, all the extracts showed considerable activity as compared to BHT $(\mathrm{p}<0.05)$.

Phenolic compounds in plants have antioxidant activity. Intensive studies on the phenolic and flavonoid constituents in Moringa oleifera have been conducted. Present study is the first to find antioxidant and antidiabetic role of saponin isolated from MO leaves. Reference to preliminary phytochemical investigation, important chemicals like alkaloids, tannins, flavonoids, saponins and terpenoids were prevalent in MO samples and these findings are agreeing with previously reports (Kasolo et al., 2010; Krishnaiah et al., 2009).

Table 1. Antioxidant profile

\begin{tabular}{llllllll}
\hline \hline Antioxidant components & ME & SF & HF & BF & CF & EF & BHT \\
\hline TPC (GAE g/100 g DW) & $12.8 \pm 2.1$ & - & $9.7 \pm 1.4$ & $10.0 \pm 1.2$ & $10.4 \pm 3.1$ & $11.6 \pm 2.4$ & - \\
TFC (CE g/100 g DW) & $9.3 \pm 2.5$ & - & $5.3 \pm 1.0$ & $6.5 \pm 1.07$ & $7.0 \pm 2.3$ & $7.8 \pm 1.9$ & - \\
DPPH, IC $50(\mu \mathrm{g} / \mathrm{mL})$ & $83.1 \pm 1.3$ & $70.4 \pm 2.1$ & $69.5 \pm 1.56$ & $72.4 \pm 1.6$ & $76.5 \pm 1.78$ & $78.1 \pm 2.34$ & $21.5 \pm 0.27$ \\
\hline \hline
\end{tabular}

Data $($ Mean $\pm \mathrm{SD})$ are average of three samples of each extract/fraction, analyzed individually in triplicate $(n=1 \times 3 \times 3)$.

$\mathrm{DW}=$ dry weight; TPC $=$ total phenolic contents expressed g gallic acid equivalent $/ 100 \mathrm{~g}$ dry weight; $\mathrm{TFC}=$ total flavonoid contents expressed as $\mathrm{g}$ catechin equivalents $/ 100 \mathrm{~g}$ dry weight; DPPH $=$ 2, 2-diphenyl 1-l-1-picrylhydrazyl, $\mathrm{ME}=$ methanol extract; $\mathrm{SF}=$ saponins fraction; $\mathrm{HF}=n$-hexane fraction; $\mathrm{BF}=n$-butanol fraction, $\mathrm{CF}=$ chloroform fraction; $\mathrm{EF}=$ ethyl acetate fraction; $\mathrm{BHT}=$ Butylated hydroxy toluene. 
Data regarding TPC and TFC was in accordance with that of the Sultana et al. (2009). They reported 9.7212.2 GAE (g/100 g dry weight) TPC and 5.3-8.66 CE ( $\mathrm{g} / 100 \mathrm{~g}$ dry weight) TFC in MO. Solubility of phytoconstituents although variable, plays a vital role in their activities. This can be due to the higher ability of polar solvents such as methanol and aqueous extract phenolics oftenly in higher amounts (Sultana et al., 2009). Another study (Kasolo et al., 2010), mentioned $45.21 \mathrm{mg} \mathrm{GAE} / \mathrm{g}$ extract and $15.39 \mathrm{mg} \mathrm{CE} / \mathrm{g}$ extract in $\mathrm{MO}$ leaves.

A very familiar trend often observed in the study of phytoconstituents is the parallel relation between total phenols and free radical scavenging power. Higher the TFC, higher will be the antioxidant efficacy observable in DPPH methodology. With free radical scavenging power, antioxidants offer defense against numerous diseases. A simple and precise approach to investigate antioxidant potential is DPPH stable free radical method (Choi et al., 2002; Koleva et al., 2002). Likewise, DPPH activity measured in present results were analogous to DPPH activity (69.5-83.1\%S) stated previously (Sultana et al., 2009). Although saponins do have antioxidant potential (DPPH scavenging by SF was not quite impressive as compared to other fractions) in fact it was lower than most of them, as it lacked the major radical scavenging components.

Antidiabetic activities. Data on antiglycation potential is summarized in Fig. 1. SF was leading inhibitor $(\mathrm{p}<0.041)$ after $\mathrm{MeOH}$ throughout the studied weeks, attaining up to $48.1 \%$ glycation restriction. Three fractions viz. $n$-hexane, $n$-butanol, chloroform demonstrated almost same though noticeable $(\mathrm{p}<0.05)$ inhibition patterns (W1: 12.86-25.71\%, W3: 34.78-42\%, W5: 44.93-47.83\%). Comparatively, there was almost 4-fold difference between the least and most potent glycation inhibitors ( $\mathrm{MeOH} v s$. ethyl acetate) in the first week of incubation. Nonetheless, this difference was lessened in the last study week. Aminoguanidine was most potent $(\mathrm{p}<0.023)$ against glycation process (W1: 44\%, W3: $62 \%$, W5: $68 \%$ ). Contrary to that, ethyl acetate fraction was least effective, as glycation inhibition was enhanced by $8.57 \%$ in $1^{\text {st }}$ week to $37.68 \%$ in the $5^{\text {th }}$ week. Significant differences $(p<0.011)$ in glycation inhibition means were evident. Follow up examinations after two and three weeks revealed that corresponding response activities for each group were positive. Diabetes mellitus and its manifestations originate from persistent hyperglycemia. Central theme in therapeutic options against such complications is to restrict high blood glucose through the mediation of natural products. Moringa oleifera is one of the renowned medicinal plants proved to have the promising effects in the prevention and treatment of diabetes through different mechanisms (Adisakwattana and Chanathong, 2011). MO leaves extract, fraction were assessed for their antiglycation ability, and competence was compared with synthetic glycation inhibitor aminoguanidine. $\mathrm{MeOH}$ repressed glycation up to $20-57 \%$ after 5 weeks. Similar to our results, Awasthi (2013) questioned the antiglycation activity of MO leaves on the basis of formation of AGEs and amadori products. The amadori and AGEs inhibitory activity was about $70 \%$ and was persistent with the incubation time. Regarding SF, antiglycation ability was higher among most of the other samples. This data can be justified by the fact that antioxidant and antiglycation activities of many medicinal plants correlate with corresponding saponin content (Bi et al., 2012; Xi et al., 2008). It was further revealed that glycation inhibition was associated with the prolonged incubation period. Similar to present inferences, Ngamukote et al. (2014) determined hindering effect of extract of M. oleifera leaf on fructose-induced glycation for 7 ,

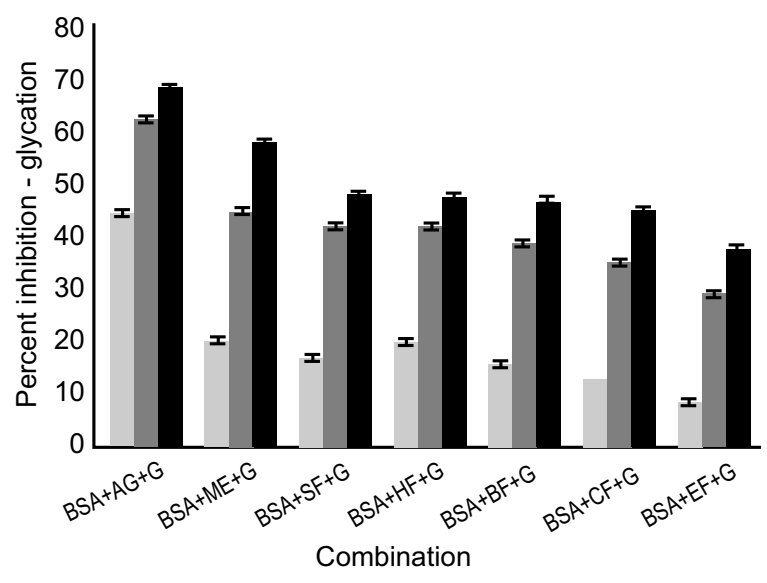

W1 W3םW5

Fig. 1. Antiglycation activity.

Data (mean $\pm \mathrm{SD}$ ) are average of three samples of each extract/fraction, analyzed individually in triplicate $(n=1 \times 3 \times 3)$. BSA $=$ Bovine serum albumin; $\mathrm{AG}=$ Aminoguanidine; $\mathrm{G}=$ Glucose; $\mathrm{ME}=$ Methanol extract; $\mathrm{SF}=$ saponins fraction; $\mathrm{HF}=n$-hexane fraction; $\mathrm{BF}=n$-butanol fraction; $\mathrm{CF}=$ chloroform fraction; $\mathrm{EF}=$ ethyl acetate fraction. Inhibition was assessed after $1^{\text {st }}$, $3^{\text {rd }}$ and $5^{\text {th }}$ weeks (W1, W3, W5) incubations. 
14, 21 and 28 days. A decline in formation of AGEs was observed with prolonged incubations.

Starch hydrolyzing enzymes inhibitors may exert some of their benefits in the therapy of diabetes mellitus. It was investigated whether alpha amylase activity could be minimized by plant extracts (Fig. 2). Negligible enzyme inhibition (3.4-9.2\%) was reflected by MO fractions, though methanol extract attained greatest (5.5-9.2\%) amylase inhibitory potential. Enzyme inhibition by SF was less impressive as compared to that of $\mathrm{MeOH}$ extract. Nearly equivalent yet nonsignificant $(p>0.198)$ percent reduction in enzyme activity by $0.1 \mathrm{~mL}, 0.3 \mathrm{~mL}$ and $0.5 \mathrm{~mL} \mathrm{SF}$ was evident, nullifying its concentration dependent impact. Concentration dependent effect of methanol extracts was more apparent $(\mathrm{p}<0.043)$ as increasing extract levels from $0.1 \mathrm{~mL}$ to $0.5 \mathrm{~mL}$ lowered the amylase activity by $3.7 \%$. Least enzyme inhibition was shown by $n$-butanol $(0.5$ $\mathrm{mL})$. Significant differences $(\mathrm{p}<0.01)$ in enzyme inhibition means were evident. Throughout the treatment period, there was a significant decrease in enzyme activities $(\mathrm{p}<0.001)$ with the magnitude of the reduction increasing with the inhibitor concentrations.

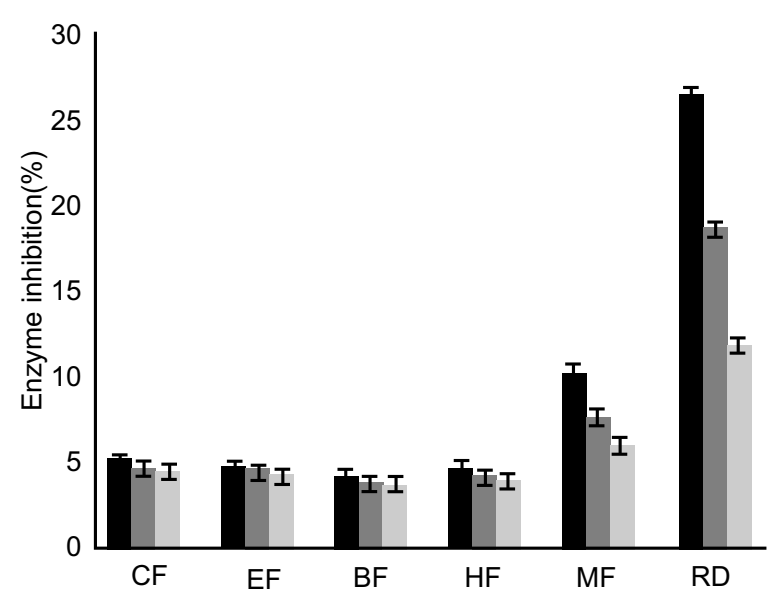

$\square 0.5 \mathrm{~mL} \square 0.3 \mathrm{~mL} \quad 0.1 \mathrm{~mL}$

Fig. 2. Alpha amylase inhibition by plant extract, fractions and standard drug.

Data (mean \pm SD) are average of three samples of each extract/fraction, analyzed individually in triplicate $(n=1 \times 3 \times 3)$. $\mathrm{RD}=$ reference drug (acarbose); $\mathrm{ME}=$ Methanol extract; $\mathrm{SF}=$ saponins fraction; $\mathrm{HF}=n$ hexane fraction; $\mathrm{BF}=n$-butanol fraction; $\mathrm{EF}=$ ethyl acetate fraction; $\mathrm{CF}=$ chloroform fraction. Inhibitions were assessed with $0.1,0.3,0.5 \mathrm{~mL}$ extracts/fractions/synthetic inhibitor volume.
All the other extracts improved their inhibition potential up to $0.6 \%$ for the same increase in corresponding concentrations. Maximum enzyme inhibitory potential was exhibited by synthetic inhibitor acarbose $(23.7 \%$, $\mathrm{p}<0.025$ ).

Using the inhibitors of carbohydrate digesting enzymes obtained from natural resources with less adverse effects than synthetic drugs can be advantageous to block the absorption of dietary carbohydrate (Etxeberria et al., 2012). Plants are important source of chemical compounds with ability of $\alpha$-amylase inhibition (Sales et al., 2012).

Enzyme inhibition by MO in present study was lesser than that calculated for other medicinal plants. Present results can be justified by the fact that an association exists between phenolic, flavonoids and tannins and the alpha amylase inhibitory activities. Studies on MO for enzyme inhibition phenomenon are scarce. Adisakwattana and Chanathong (2011) documented slight inhibitory potential of pancreatic alpha amylase by MO leaves extracts. Amongst the phytoconstituents investigated, flavonoids demonstrated maximum inhibitory potential related to the number of hydroxyl groups present in molecules of the compound (Sales et al., 2012). SF showed negligible amylase inhibition being devoid of flavonoids. Triterpenoids represent a favourable and expanding natural source of biologically active constituents. Despite their wide distribution in plants, investigations on inhibition of activities of alpha amylase and alpha glucosidase are limited. A few reports available to date dearth the mechanism by which this activity occurs (Sales et al., 2012; Luo et al., 2008). Multiple comparisons of means and inter-dependence of weekly analysis showed significant associations for glycation assay. Similarly, enzyme inhibition improved after increasing inhibitor concentrations. It is assumed that saponins existing in MO leaves hold antioxidant, antiglycation and enzyme inhibitory activities that can lead to the development of innovative therapeutic adjuvants. However, despite some promising results, more research is needed to validate current inferences.

Conflict of interest. The authors declare no conflict of interest.

\section{References}

Adisakwattana, S., Chanathong, B. 2011. Alphaglucosidase inhibitory activity and lipid-lowering mechanisms of Moringa oleifera leaf extract. 
European Review for Medical and Pharmacological Sciences, 15: 803-808.

Ahmad, S., Shah, S.M., Alam, M.K., Usmanghani, K., Azhar, I., Akram, M. 2014. Antipyretic activity of hydro-alcoholic extracts of Moringa oleifera in rabbits. Pakistan Journal of Pharmaceutical Sciences, 27: 931-934.

Akanni, E.O., Adedeji, A.L., Adedosu, O.T., Olaniran, O.I., Oloke, J.K. 2014. Chemopreventive and antileukemic effects of ethanol extracts of Moringa oleifera leaves on wistar rats bearing benzene induced leukemia. Current Pharmaceutical Biotechnology, 15: 563-568.

Apostolidis, E., Kwon, Y.I., Shetty, K. 2006. Potential of cranberry based herbal synergies for diabetes and hypertension management. Asia Pacific Journal of Clinical Nutrition, 15: 433-441.

Astuti, S.M., Sakinah, M., Andayani, R., Risch, A. 2011. Determination of saponin compound from Anredera cordifolia (Ten) steenis plant (Binahong) to potential treatment for several diseases. Journal of Agricultural Science, 3: 224-232.

Awasthi, S. 2013. Understanding the mechanism of antidiabetic activity and efficacy of functional foods against advanced glycation end products: Nigella sativa and Moringa oleifera. Planta Medica, 79: PN8.

Ayatollahi, S.A.M., Kobarfard, F., Asgarpanah, J., Choudhary, M.I. 2010. Antiglycation activity of Otostegia persica (Burm.) Boiss. African Journal of Biotechnology, 9: 3645-3648.

Bharali, R., Tabassum, J., Azad, R. 2003. Chemomodulatory effect of Moringa oleifera, Lam, on hepatic carcinogen metabolising enzymes, antioxidant parameters and skin papillomagenesis in mice. Asian Pacific Journal of Cancer Prevention, 4: 131-139.

Bi, L., Tian, X., Dou, F., Hong, L., Tang, H., Wang, S. 2012. New antioxidant and antiglycation active triterpenoid saponins from the root bark of Aralia taibaiensis. Fitoterapia, 83: 234-240.

Chahardehi, A.M., Ibrahim, D., Suleman, S.F. 2009. Antioxidant activity and polyphenolic contents of some medicinal plants in Urticaceae family. Journal of Biological Sciences, 3: 25-29.

Choi, C.W., Kim, S.C., Hwang, S.S., Choi, B.K., Ahn, H.J., Lee, M.Y., Park, S.H., Kim, S.K. 2002. Antioxidant activity and free radical scavenging capacity between Korean medicinal plants and flavonoids by assay-guided comparison. Plant Science, 163: 1161-1168.
Etxeberria, U., DeLa Garza, L.A., Campion, J., Martinez, J.A., Milagro, F.I. 2012. Antidiabetic effects of natural plant extracts via inhibition of carbohydrate hydrolysis enzymes with emphasis on pancreatic alpha amylase. Expert Opinion on Therapeutic Targets, 16: 269-297.

Jung, I.L. 2014. Soluble extract from Moringa oleifera leaves with a new anticancer activity. PLoS One, 9: e95492.

Kasolo, J.N., Bimenya, G.S., Ojok, L., Ochieng, J., Ogwal-okeng, J.W. 2010. Phytochemicals and uses of Moringa oleifera leaves in Ugandan rural communities. Journal of Medicinal Plant Research, 4: 753-757.

Kaur, A., Kaur, P.K., Singh, S., Singh, I.P. 2014. Antileishmanial compounds from Moringa oleifera Lam. Zeitschrift für Naturforschung C, 69: 110116.

Koleva, I.I., Van Beek, T.A., Linssen, J.P.H., De Groot, A., Evstatieva, L.N. 2002. Screening of plant extracts for antioxidant activity: A comparative study on three testing methods. Phytochemical Analysis, 13: 8-17.

Koneri, R.B., Samaddar, S., Ramaiah, C.T. 2014. Antidiabetic activity of a triterpenoid saponin isolated from Momordica cymbalaria Fenzl. Indian Journal of Experimental Biology, 52: 46-52.

Krishnaiah, D., Devi, T., Bano, A., Sarbatly, R. 2009. Studies on phytochemical constituents of six malaysian medicinal plants. Journal of Medicinal Plants Research, 3: 067-072.

Kurmi, R., Ganeshpurkar, A., Bansal, D., Agnihotri, A., Dubey, N. 2014. Ethanol extract of Moringa oliefera prevents in vitro glucose induced cataract on isolated goat eye lens. Indian Journal of Ophthalmology, 62: 154-157.

Luo, J.G., Ma, L., Kong, L.Y. 2008. New triterpenoid saponins with strong a-glucosidase inhibitory activity from the roots of Gypsophila oldhamiana. Bioorganic \& Medicinal Chemistry, 16: 2912-2920.

Minaiyan, M., Asghari, G., Taheri, D., Saeidi, M., NasrEsfahani, S. 2014. Anti-inflammatory effect of Moringa oleifera Lam. seeds on acetic acid-induced acute colitis in rats. Avicenna Journal of Phytomedicine, 4: 127-136.

Ngamukote, S., Nunthanawanich, P., Adisakwattana, P.S., Mkynen, K., Dahlan, W. 2014. The protective effect of Moringa oleifera leaf extract on fructosemediated protein glycation in vitro (829.10). The FASEB Journal, 28: 829.10. 
Rao, A.V., Gurfinkel, D.M. 2000. The bioactivity of saponins: Triterpenoid and steroidal glycosides. Drug Metabolism and Drug Interactions, 17: 211-235.

Sahoo, S., Raghavendra, K.M., Biswas, S. 2014. Identification of a proteinaceous component in the leaf of Moringa oleifera Lam. with effects on high serum creatinine. Indian Journal of Pharmaceutical Sciences, 76: 78-81.

Sales, P.M., Souza, P.M., Simeoni, L.A., Silveira, D. 2012. $\alpha$-amylase inhibitors: A review of raw material and isolated compounds from plant source. Journal of Pharmacy and Pharmaceutical Sciences, 15: 141-183.

Sangkitikomol, W., Rocejanasaroj, A., Tencomnao, T. 2014. Effect of Moringa oleifera on advanced glycation end-product formation and lipid metabolism gene expression in HepG2 cells. Genetics and Molecular Research, 13: 723-725.

Siddique, N.A., Mujeeb, M., Najmi, A.K., Akram, M. 2010. Evaluation of antioxidant activity, quantitative estimation of phenols and flavonoids in different parts of Aegle marmelos. African Journal of Plant Sciences, 4: 001-005.

Souri, E.G., Amin, G., Farsan, H., Jalalizadeeh, H., Barezi, S. 2010. Screening of thirteen medicinal plants extracts for antioxidant activity. Iranian Journal of Pharmaceutical Research, 7: 149-154.
Sultana, B., Anwar, F., Ashraf, M. 2009. Effect of extraction solvent/technique on the antioxidant activity of selected medicinal plant extracts. Molecules, 14: 2167-2180.

Sun, H.X., Xie, Y., Ye, Y. 2009. Advances in saponinbased adjuvants. Vaccine, 27: 1787-1796.

Thao, N.P., Luyen, B.T., Jo, S.H., Hung, T.M., Cuong, N.X., Nam, N.H., Kwon, Y.I., Minh, C.V., Kim, Y.H. 2014. Triterpenoid saponins from the roots of Rosa rugosa Thunb. as rat intestinal sucrase inhibitors. Archives of Pharmacal Research, 37: 1280-1285.

Wagner, R., Bladt, S. 1996. Plant Drug Analysis, A Thin Layer Chromatography Atlas. $2^{\text {nd }}$ edition, Springer, Berlin, Germany.

Waterman, C., Cheng, D.M., Rojas-Silva, P., Poulev, A., Dreifus, J., Lila, M.A., Raskin, I. 2014. Water extractable isothiocyanates from Moringa oleifera leaves attenuate inflammation in vitro. Phytochemistry, 103: 114-122.

Xi, M., Hai, C., Tang, H., Chen, M., Fang, K., Liang, X. 2008. Antioxidant and antiglycation properties of total saponins extracted from traditional Chinese medicine used to treat diabetes mellitus. Phytotherapy Research, 22: 228-237.

Yassa, H.D., Tohamy, A.F. 2014. Extract of Moringa oleifera leaves ameliorates streptozotocin-induced diabetes mellitus in adult rats. Acta Histochemical, 116: $844-854$. 